\title{
Mass transport properties of the tetrahydronaphthalene/n-dodecane mixture measured by investigating non-equilibrium fluctuations
}

\author{
Fabrizio Croccolo ${ }^{\mathrm{a}, *}$, Frank Scheffold ${ }^{\mathrm{a}}$, Henri Bataller ${ }^{\mathrm{b}}$ \\ a Department of Physics, University of Fribourg, chemin du Musée 3, CH-1700 Fribourg, Switzerland \\ ${ }^{\mathrm{b}}$ Laboratoire des Fluides Complexes et leurs Réservoirs - UMR 5150, Université de Pau et des Pays de l'Adour, BP 1155,64013 Pau cedex, France
}

\begin{abstract}
We present preliminary near-field light scattering (NFS) data concerning the analysis of the static power spectrum and of the relaxation time constant as a function of the wave vector for non-equilibrium fluctuations (NEFs). The goal of these measurements is to obtain information about the Soret and the mass diffusion coefficients of a binary mixture undergoing thermodiffusion. In particular, we show how the interaction between NEFs and the gravity force gives rise to a critical wavelength that provides additional information about the Soret coefficient. We suggest that a quantitative analysis can be performed by means of this non-invasive optical technique. In our setup, the sample is monitored parallel to the imposed temperature gradient, thus being insensitive to the refractive index profile along the vertical axis, while at the same time we are able to detect the light scattered by the refractive index fluctuations in horizontal planes. We select a shadowgraph layout for the NFS setup due to the extremely small wave vectors we aim to analyze. From a double-frame differential analysis of the acquired images, we obtain both the static power spectrum and the dynamics of NEFs. As a proof-of-principle experiment, we present Soret and diffusion coefficient data on a liquid mixture of tetrahydronaphthalene $/ n$-dodecane.
\end{abstract}

\section{Introduction}

The molecules of a multi-component fluid mixture tend to separate upon the application of a temperature gradient, which is known as the Ludwig-Soret, or simply Soret effect [1,2]. The scientific community happens to be aware of the existence of the Soret effect since more than 150 years; nevertheless, the actual microscopic mechanism of how and why the molecules move still remains not completely understood. This is unfortunate, not least given the practical implications of the Soret effect. Oil companies, for example, are very much interested in including the Soret effect into their modeling tools to predict how the composition of oil-rich mixtures in underground fields is distributed. Actually, different theories have been suggested to describe some of the features of the Soret effect [3-8] and moreover a large number of studies have addressed the problem by numerical simulations [9-12]. A variety of experimental methods have been applied to characterize the Soret effect [13-20]. However, a much better theoretical and experimental understanding of the Soret effect is still needed in order to facilitate detailed predictions of the behavior of multi-component fluids. It is noteworthy that many studies of multi-component mixtures involve micro-gravity conditions in order to avoid convection, thus substantially complicating the experiments [21-23].

\footnotetext{
* Corresponding author.

E-mail address: fabrizio.croccolo@univ-pau.fr (F. Croccolo).
} 
The quantification of the Soret effect is mainly related to the ratio between the applied temperature gradient and the steady-state concentration gradient, leading to the well-known empirical definition of the Soret coefficient $S_{T}$ :

$$
S_{T}=-\frac{1}{c_{0}\left(1-c_{0}\right)} \frac{\nabla c}{\nabla T}
$$

Up to now, most techniques for measuring the Soret coefficient involved analysis of the vertical concentration profile, while a (typically) binary mixture is subjected to a temperature gradient in the vertical direction. For example, the beam deflection technique [24-26] analyses the deflection of a laser beam due to the refractive index gradient that develops within the sample because of both the temperature and the concentration gradient. In this case, the vertical displacement of the beam at a given distance from the cell output window carries information about the refractive index gradients within the cell. From the analysis of this displacement as a function of time, an accurate measurement of the diffusion and Soret coefficient can be obtained. In this case, to extract numerical values of these two quantities, one needs to know the dependence of the refractive index upon temperature and concentration, i.e. the so-called contrast factors of the analyzed mixture [27, 28]. An alternative method that is not based on an optical analysis is the thermo-gravitational column technique [29], in which the sample is let evolve to the steady state of the separation, after which small volumes of the fluid mixture at different heights are physically picked-up and analyzed in order to directly measure the value of the concentration as a function of the vertical coordinate, thus leading to a measurement of the thermodiffusion coefficient $D_{T}$, but without any information about the mass diffusion coefficient $D$. Other interesting techniques from the optical domain include optical digital interferometry (ODI) [30], thermal diffusion-forced Rayleigh scattering (TDFRS) [31], thermal lens [32] and other techniques that have been reported in the literature [17,28,33-35].

In this paper we present preliminary data on how the mass diffusion and the Soret coefficients can be effectively measured by means of careful investigation of the static and dynamic power spectra of non-equilibrium fluctuations (NEFs) occurring while a binary mixture is stressed by a thermal gradient in the presence of a gravitational field. The optical technique utilized here is that of dynamic near field scattering with a shadowgraph setup.

The remainder of this paper is organized as follows: in Section 2 the experimental setup is described, providing details of the thermodiffusion cell, the shadowgraph setup, and the experimental optical procedure, including differential dynamic analysis, in Section 3 the theory of non-equilibrium fluctuations is summarized to provide the working equations used in the following, in Section 4 experimental results are presented and compared with literature data, and finally conclusions are provided in Section 5.

\section{Experimental setup and procedure}

\subsection{Experimental setup}

The core of the experimental apparatus is a thermodiffusion cell, specifically designed for applying a temperature gradient to a thin slab of a multi-component fluid. In Fig. 1, the whole apparatus, consisting of the thermodiffusion cell, the temperature control, and the optical setup, is sketched. The thermodiffusion cell is designed for applying a thermal gradient to a thin horizontal layer of fluid and providing optical access to a central area while maintaining an excellent thermal homogeneity and stability. The cell core consists of two sapphire square plates with side $a=40 \mathrm{~mm}$ and height $b=8 \mathrm{~mm}$, which are put one on top to the other and maintained at the desired distance by four spacers of the desired height $h$ (in the present work, we report experiments for $h=1.53 \mathrm{~mm}$ ). The fluid is horizontally confined by a Viton O-ring pressed by the two sapphire windows. The faces of the sapphire plates external to the cell are in thermal contact with two aluminum plates, where two thermistors (Wavelenght Electronics, TCS651) are installed to constantly monitor the temperature of the two sapphire windows. External to the two aluminum plates there are two Peltier elements (Kryotherm, TB-109-1.4-1.5 CH), which provide the temperature control, regulated by two temperature controllers (TCs) (Wavelenght Electronics, LFI-3751). At the end, at the external faces of the Peltier elements, two aluminum plates are flown with water coming from a thermostatic bath to remove the excess heat produced by the Peltier elements.

The temperature control system allows us to separately set the temperatures of the two opposite sides of the cell with a temperature stability better than $1 \mathrm{mK}$ RMS over one day. The absolute values of the measured temperatures were calibrated with a thermometer prior to the measurements with a precision of $\pm 0.02 \mathrm{~K}$. The cell is filled with two syringe needles through the Viton O-ring. During the filling procedure, the cell is inclined and the fluid is pushed through the bottom syringe, while air is removed through the top one. After filling, the two needles are carefully removed and the holes in the O-ring close due to the pressure exerted on it by the sapphire windows. Attention is paid to avoid air bubbles in the filling procedure.

The optical technique we employed is known as Near-Field Scattering [36-45] by means of a shadowgraph setup [46-48]. The shadowgraph optical setup involves a low coherence light source (Super Lumen Diodes, Broad Lighter S680) connected with a single-mode fiber whose output is collimated by an achromatic doublet lens $(f=15 \mathrm{~cm}, \phi=5 \mathrm{~cm})$. The light polarization is set by a fixed linear polarizer just after the collimating lens. A second motorized polarizer after the sample allows remote control of the intensity of the light impinging onto the charged coupled device (CCD) camera (Vosskühler, CCD4000) positioned at a distance $z=26 \mathrm{~cm}$ from the sample plane. The camera sensor consists of $2048 \times 2048$ square pixels with a side of $7.4 \mu \mathrm{m}$. No optics is installed between the sample cell and the CCD sensor. The camera is able to 


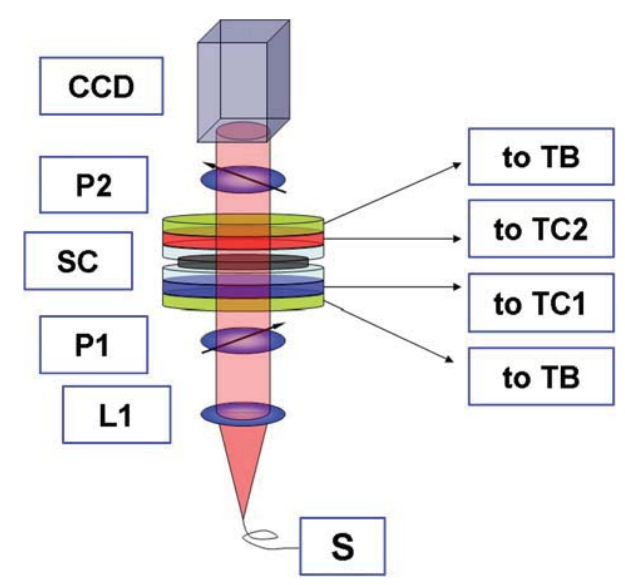

Fig. 1. Sketch of the experimental setup: light coming from the optical source (S) impinges on a collimating lens (L1), after which it passes through a polarizer (P1), the sample cell (SC), an analyzer (P2), and finally impinges onto the pixilated detector (CCD). The sample is contained horizontally within an O-ring (dark slab) and vertically within two sapphire plates (cyan) which are thermally controlled by two Peltier elements (blue and red) which are electrically driven by two thermo-controllers (TC1 and TC2); the excess heat is sinked into the thermostatic bath (TB). Please refer to the online version for interpretation of colors.

transfer about 4 images per second to the PC connected via a Camera Link connection. In order to save the images as quickly as possible, storage is made on a solid state disk (SSD).

\subsection{Dynamic near-field imaging}

After acquiring a series of images, scattering information is extracted by means of statistical analysis of the image Fourier transforms. In this case, we apply double-frame differential analysis $[49,47,46,50-52]$ in order to extract both the static structure factor and the temporal correlation function. Since this processing requires a heavy computational effort, we make use of custom-made software in the CUDA/C++ language, taking advantage of the parallelization capabilities of a graphic board (Nvidia, TESLA C2050). Details of the architecture of this software and its performance are described in a separate publication [50].

Images acquired by means of a near-field scattering setup consist of an intensity map $I(\vec{x}, t)$ generated by the interference on the CCD plane between the portion of the incident beam that has passed undisturbed through the sample and the beams scattered by refractive index fluctuations occurring within the sample. Statistical analysis involving fast Fourier transforms (FFTs) provides accurate measurements of the intensity $I_{S}(\vec{q}, t)$ of the light scattered at each wave vector $q$ grabbed by the optical setup and for all the times $t$ of the acquisition sequence. Different setups show different responses to the acquired signal as a function of the wave vector $q$, which is described by the so-called transfer function $T(q)$ of the technique. It has been shown that the shadowgraph transfer function dependence upon the wave vector shows a sinusoidal behavior $[48,53,54]$ :

$$
T(q)=\sin ^{2}\left(\frac{q^{2} z}{2 k_{0}}\right)
$$

where $z$ is the visualization distance and $k_{0}$ is the wave vector of the incident light in vacuum. Eq. (2) is valid for an infinitely thin sample at a distance $z$ from the plane imaged onto the CCD camera (in the present setup, the latter coincides with the sample plane, since no optics is placed after the sample). In the case of a Soret experiment, both temperature and concentration gradients are distributed over the entire height of the cell, such that the sample thickness is not negligible. Unfortunately, no quantitative theory currently describes the transfer function of a shadowgraph setup under such conditions. We find a satisfactory match between our experimental data and an empirical equation able to take into account the progressive disappearance of the shadowgraph oscillations due to the finite sample thickness:

$$
T(q)=\frac{1}{2}+\left[\sin ^{2}\left(\frac{q^{2} z}{2 k_{0}}\right)-\frac{1}{2}\right]\left[\exp -\left(\frac{q}{q_{\text {thick }}}\right)^{\gamma}\right]
$$

where $q_{\text {thick }}$ is a typical wave vector related to the disappearance of transfer function oscillations and $\gamma$ is an exponent describing how fast the oscillations are washed out. Although there is no theoretical basis for the empirical equation (3), we find the quality of the resulting fits excellent, providing robust estimates of the system parameters, which is not the case when using Eq. (2), for example. The dynamic near-field imaging technique has been shown to provide direct access to the structure function of the sample for all the wave vectors in the optical system by means of a statistical analysis of differences of images. Details of the dynamic analysis can be found elsewhere $[46,47,49,50]$. Here we just recall the working equation that is used to fit experimental data: 


$$
G(q, \mathrm{~d} t)=\left\langle\left|\Delta I_{\mathrm{m}}(q, \mathrm{~d} t)\right|^{2}\right\rangle=2 a\left\{T(q) I_{\mathrm{s}}(q)[1-f(q, \mathrm{~d} t)]+B(q)\right\}
$$

where $G(q, \mathrm{~d} t)$ is the structure function for the given wave vector $q$ and time delay $\mathrm{d} t, \Delta I_{\mathrm{m}}(q, \mathrm{~d} t)$ is the Fourier transform of the difference of two images with a time delay $\mathrm{d} t, I_{\mathrm{s}}(q)$ is the intensity of the light scattered by the sample, $f(q, \mathrm{~d} t)$ is the intermediate scattering function and $B(q)$ is the background noise of the measurement. For many cases of practical interest, the intermediate scattering function can be written as an exponential decay $f(q, \mathrm{~d} t)=\exp (-\mathrm{d} t / \tau)$; in this case, Eq. (4) takes the form:

$$
G(q, \mathrm{~d} t)=\left\langle\left|\Delta I_{\mathrm{m}}(q, \mathrm{~d} t)\right|^{2}\right\rangle=2 a\left\{T(q) I_{\mathrm{s}}(q)[1-\exp (-\mathrm{d} t / \tau(q))]+B(q)\right\}
$$

Fitting the structure function by means of Eq. (5) with $T(q) I_{s}(q), \tau(q)$ and $B(q)$ as free fitting parameters for each wave vector allows the determination of the static power spectrum $T(q) I_{S}(q)$ and the time constant $\tau(q)$. It is also worth pointing out that in contrast to a conventional static NFS experiment, this approach enables us to obtain the background noise $B(q)$ without performing a separate measurement. The double-frame differential dynamics approach has proven to be very useful and has already been applied in many other realizations of the NFS technique [51,52,55-58].

\section{Non-equilibrium fluctuations}

Non-equilibrium fluctuations (NEFs) are tiny (in intensity) but giant (in lateral size) fluctuations of the thermodynamic variables that are always tied to diffusive processes and therefore to gradients. It is a common feature of physical processes that the local value of a thermodynamic variable fluctuates around a certain mean value. This happens also under equilibrium conditions and, for example, the fluctuations of the refractive index are the reason why a homogeneous pure fluid scatters light. Under non-equilibrium conditions, the gradient of one variable couples the spontaneous fluctuations of the velocity of the fluid molecules with the fluctuations of the relevant variable, thus providing a huge enhancement of the intensity of the fluctuations, increasing with their size. This phenomenon has been widely investigated in the latest decades and a sound theoretical description (see for example [59-63]) as well as many experimental studies [46,47,49,64-67] can be found in the literature. Here, we would like to recall the essential equations that describe the intensity of the light scattered by concentration fluctuations generated in a fluid out of equilibrium and in the presence of the terrestrial gravitational field. The intensity of the scattered light $I_{s}(q)$ as a function of the wave vector $q=2 \pi / \lambda$ (where $\lambda$ is the fluctuation lateral dimension) shows a power-law behavior for wave vectors larger than a critical value $q_{\mathrm{c}}$, and a plateau for smaller wave vectors. This is well described by the relation:

$$
I_{S}(q)=\frac{I_{0}}{1+\left(q / q_{\mathrm{c}}\right)^{4}}
$$

where $I_{\mathrm{S}}(q)$ is the static power spectrum, $I_{0}$ is the plateau value, and the critical wave vector $q_{\mathrm{c}}$ can be demonstrated to be related to fluid parameters and the gravitational field:

$$
q_{\mathrm{c}}=\left(\frac{\beta g \nabla c}{v D}\right)^{1 / 4}
$$

where $\beta=1 / \rho(\partial \rho / \partial c)$ is the solutal expansion coefficient, $g$ is the gravitational acceleration, $\nabla c$ is the concentration gradient, $v$ is the kinematic viscosity and $D$ is the mass diffusion coefficient. The physical meaning of the critical wave vector is actually that of distinguishing the fluctuations whose behavior is mainly diffusive (large wave vectors, $q \gg q_{\mathrm{c}}$, i.e. small dimensions) from those that are mainly driven by the buoyancy force of gravity (small wave vectors, $q \ll q_{\mathrm{c}}$, i.e. large dimensions).

The influence of gravity has also been checked in micro-gravity conditions where the $q^{-4}$ dependence of the intensity is observed for smaller wave vectors than in normal gravity conditions, as can be derived from Eq. (7) $[68,69]$.

From the dynamical point of view, the temporal correlation function of NEFs are expected to follow an exponential decay for all wave vectors, with time constants $\tau(q)$ varying as a function of $q$. In fact, for wave vectors larger than the critical value, the time constant of the temporal correlation function is the well-known diffusive one $\tau(q)=1 /\left(D q^{2}\right)$, while for very small wave vectors the buoyancy force leads to a square dependence of the time constant with respect to the modulus of the wave vector $\tau(q)=q^{2} v /(\beta g \nabla c)$. Over the entire wave vector range, the relaxation time constant is given by a characteristic bell-shaped curve:

$$
\tau(q)=\frac{1}{D q^{2}\left[1+\left(q_{\mathrm{c}} / q\right)^{4}\right]}
$$

Similar reasoning can be applied also to thermal fluctuations, leading to analogous equations. Here, however, we are interested in the analysis of the concentration components, the thermal ones being too fast to be analyzed with our optical setup. Concentration NEFs in a thermodiffusion experiment are due to the Soret effect, which in turn is directly related to the fluid thermal properties (see Eq. (1)). Therefore, the critical wave vector can be written in terms of the applied thermal gradient as:

$$
q_{\mathrm{c}}=\left(\frac{\beta g \nabla T S_{T} c_{0}\left(1-c_{0}\right)}{\nu D}\right)^{1 / 4}
$$




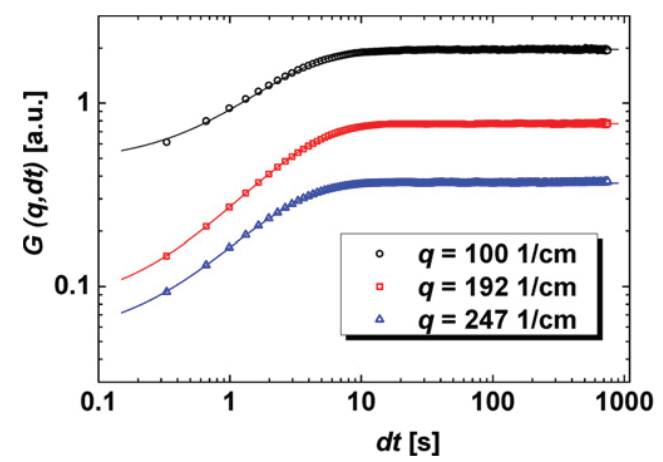

Fig. 2. Structure function $G(q, \mathrm{~d} t)$ vs. delay time $\mathrm{d} t$ for different values of the wave vector $q$ : $100 \mathrm{~cm}^{-1}, 192 \mathrm{~cm}^{-1} \mathrm{and}_{247 \mathrm{~cm}}^{-1}$ (from top to bottom). Continuous lines indicate the relative fitting functions. Data points refer to the following experimental conditions: sample of $\mathrm{THN} / n-\mathrm{C} 12, c_{0}=50 \% \mathrm{w} / \mathrm{w}$ $T_{\text {top }}=33^{\circ} \mathrm{C}, T_{\text {bottom }}=27^{\circ} \mathrm{C}$, and $\Delta t=35 \mathrm{~ms}$.

By inverting Eq. (9), one can derive an expression of the Soret coefficient $S_{T}$ as a function of the (measurable) critical wave vector $q_{\mathrm{c}}$, of the mass diffusion coefficient $D$ and of the other (known) fluid properties:

$$
S_{T}=\frac{q_{\mathrm{c}}^{4} \nu D h}{\beta g \Delta T c_{0}\left(1-c_{0}\right)}
$$

\section{Results and discussion}

A series of experimental runs with different temperature gradients $(\Delta T=8,12,16,20 \mathrm{~K})$ have been performed with a binary mixture of $1,2,3,4$ tetrahydronaphthalene and $n$-dodecane (THN $/ n$-C12) at concentration $c_{0}=50 \% \mathrm{w} / \mathrm{w}$. The sample consists of a layer of $h=1.53 \mathrm{~mm}$ and a lateral size given by the containing O-ring internal diameter of about $\phi=26 \mathrm{~mm}$. For each experimental condition, different image acquisition runs have been performed with three different acquisition times $(\Delta t=0.35,3.5,35 \mathrm{~ms})$ of the CCD sensor. Each set consisted in 3000 images taken at approximately $\mathrm{d} t_{\mathrm{min}}=250 \mathrm{~ms}$ delay time between two successive images (the total measuring time for each run thus being $750 \mathrm{~s}$ ). Each dataset has then been processed on a dedicated PC with a custom-made CUDA/C++ software in order to obtain a fast parallel processing of the images to get the structure functions for all the wave vectors and for all the time delays accessible within the image dataset [50]. The temperature gradient is applied at the beginning of each run via the two distinct temperature controllers, so that a linear temperature gradient sets up in some tens of seconds. The image acquisition is started about two hours later to be sure that the concentration gradient due to the Soret effect is fully developed.

\subsection{Analysis of non-equilibrium fluctuations}

In Fig. 2 we present three experimental structure functions as a function of the time delay between images for three different wave vectors. One can easily detect the different amplitudes set by the term $T(q) I_{S}(q)$, the different relaxation time constants $\tau(q)$ and the different starting values for $\mathrm{d} t \rightarrow 0$ given by the background noise $B(q)$. Structure functions are fitted with Eq. (5) with $T(q) I_{S}(q), \tau(q)$ and $B(q)$ as fitting parameters for any value of the wave vector $q$, as indicated by continuous lines in Fig. 2.

Fig. 3 shows the static power spectrum as obtained by fitting our data with Eq. (5). Fig. 3(a) shows the static structure factor $I_{\mathrm{S}}(q)$ of non-equilibrium fluctuations multiplied by the oscillating shadowgraph transfer function $T(q)$ together with the result of the fit with Eq. (3) and Eq. (6). The excellent quality of the fit is evident in the graph. As a further check we divided our data by the transfer function $T(q)$ (Eq. (3)) using the previously obtained values of $q_{\text {thick }}$ and $\gamma$. This result is shown in Fig. 3(b). We now clearly observe the main features of the power spectrum $I_{S}(q)$, namely the presence of a plateau for wave vectors smaller than $q_{\mathrm{c}}$ and the $q^{-4}$ decrease for larger wave vectors. The data of the structure factor can now be plotted again together with Eq. (6) in order to get an estimate of $q_{\mathrm{c}}$ and $I_{0}$. Note that in Fig. 3(b) some of the data points, very close to the minima of the shadowgraph transfer function, had to be removed due to the enhanced contribution of background noise.

In Fig. 4 the time constant $\tau(q)$ is shown as a function of the wave vector $q$ together with the fit by Eq. (8) (solid line). Again, in Fig. 4, data close to the minima in the shadowgraph transfer function have not been taken into account. It is worth pointing out again that the deviation from the diffusive behavior is related to the effect of gravity on larger size fluctuations (smaller wave vectors). This deviation generates the characteristic bell-shape of $\tau(q)$, which has already been observed and studied in a number of experiments addressing concentration non-equilibrium fluctuations in a free-diffusion experiment $[46,47,49]$. 

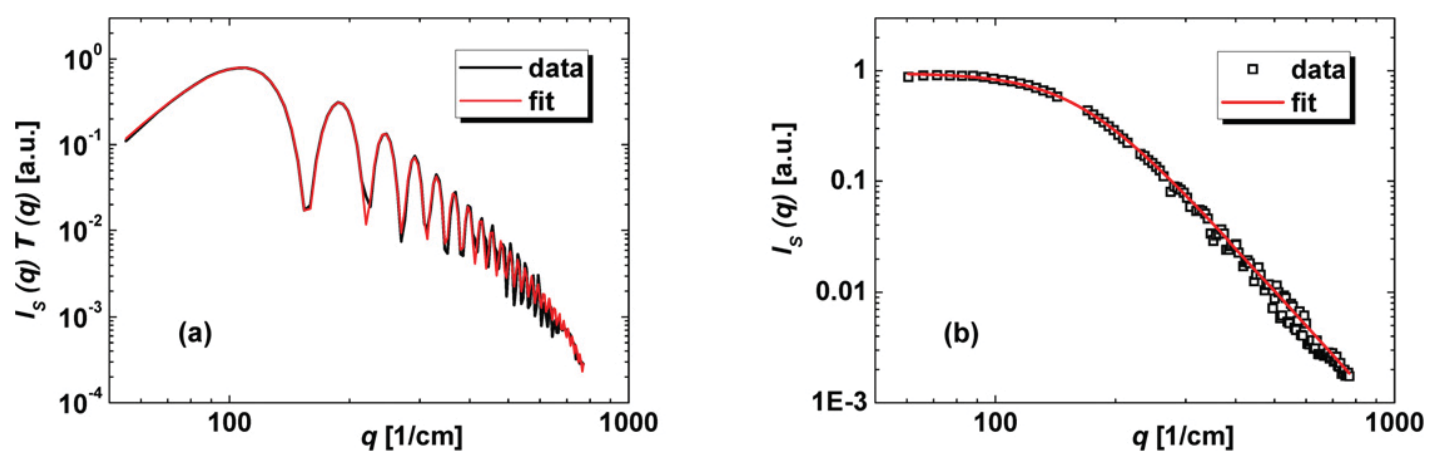

Fig. 3. (a) Static power spectrum of NEFs multiplied by the shadowgraph transfer function $T(q) I_{\mathrm{S}}(q)$ vs. wave vector $q$, and (b) static power spectrum after dividing data from Fig. 3(a) by the shadowgraph transfer function $T(q)$ as per Eq. (3) with parameters $z$, $q_{\text {thick }}$, and $\gamma$ obtained by the fitting shown in Fig. 3(a). Data points refer to the same experimental conditions as in Fig. 2.

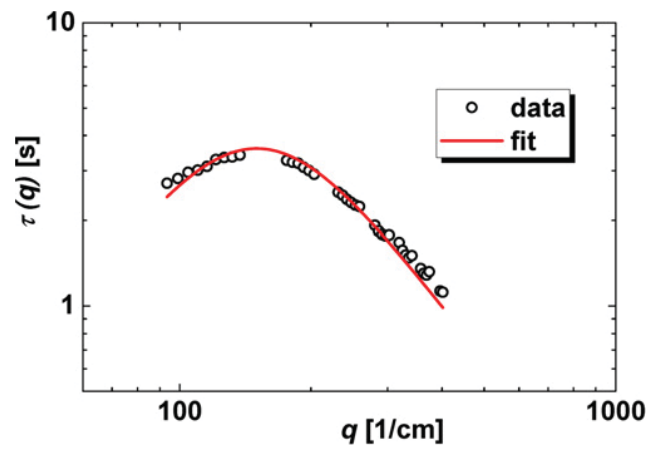

Fig. 4. Time constant $\tau(q)$ vs. wave vector $q$ : black circles are for experimental data, whereas the red (in the online version) line is for the fitting function as per Eq. (8). Data points refer to the same experimental conditions as in Fig. 2.

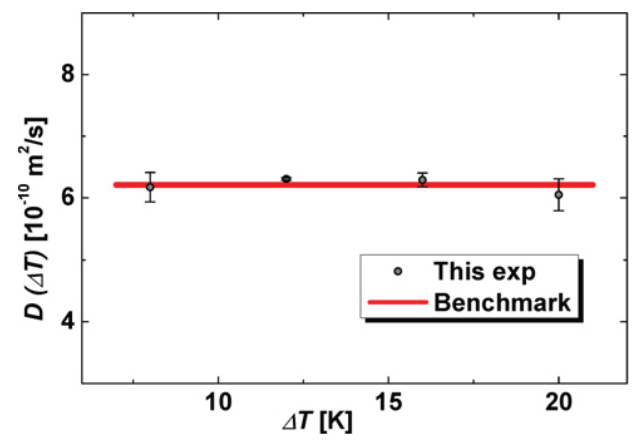

Fig. 5. Diffusion coefficient $D$ vs. applied temperature difference $\Delta T$ for the THN $/ n-C 12, c_{0}=50 \% \mathrm{w} / \mathrm{w}$ mixture at an average temperature of $25^{\circ} \mathrm{C}$ : circles are for experimental data, whereas the red (in the online version) line stands for the benchmark value of Ref. [71].

\subsection{Soret and mass diffusion coefficient}

From the analysis presented in the previous section, one can extract the values of the diffusion coefficient $D$ and of the critical wave vector $q_{\mathrm{c}}$. While the diffusion coefficient can be extracted from the analysis of the time constant only, in principle the critical wave vector can be obtained both from the statics (Eq. (6)) and from the time constants (Eq. (8)). However, our experiments indicate systematically higher values for $q_{\mathrm{c}}$ if derived from the static scattering results. A detailed analysis of this interesting finding will be presented elsewhere [70]. Here we restrict our discussion to a consistent analysis of the results obtained by the dynamics only (Fig. 4). Based on the knowledge of these two characteristic quantities, an estimate of the Soret coefficient $S_{T}$ can be derived via Eq. (10). In the following, we discuss the obtained values for $D$ and $S_{T}$ and compare it against literature data [71].

In Fig. 5 the resulting values for the diffusion coefficient of the THN/n-C12 mixture at average temperature of $25^{\circ} \mathrm{C}$ are shown as a function of the applied temperature difference. Since no dependence on the applied temperature gradient is detectable, we average the values for all the measurements and obtain a mean value of $D=(6.30 \pm 0.14) \times 10^{-10} \mathrm{~m}^{2} / \mathrm{s}$. 


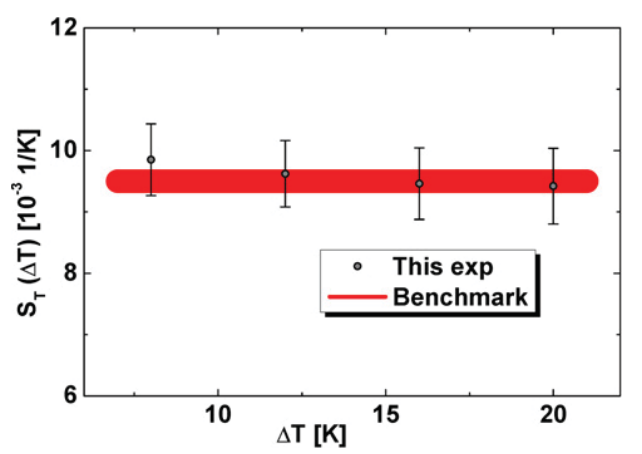

Fig. 6. Soret coefficient $S_{T}$ vs. applied temperature difference $\Delta T$ for the $\mathrm{THN} / n-\mathrm{C} 12, c_{0}=50 \% \mathrm{w} / \mathrm{w}$ mixture at an average temperature of $25^{\circ} \mathrm{C}$ : circles are for experimental data, whereas the red (in the online version) line stands for the benchmark value of Ref. [71].

In Fig. 6, the resulting values for the Soret coefficient of the THN $/ n-\mathrm{C} 12$ mixture at an average temperature of $25^{\circ} \mathrm{C}$ are shown as a function of the applied temperature difference. The average of all the measured values provides a mean value of $S_{T}=(9.59 \pm 0.13) \times 10^{-3} \mathrm{~K}^{-1}$. Finally, we are able to determine the value of the thermodiffusion coefficient $D_{T}$ as the product of the obtained values of the Soret coefficient $S_{T}$ and the mass diffusion coefficient $D$, providing $D_{T}=$ $(6.04 \pm 0.22) \times 10^{-12} \mathrm{~m}^{2} /(\mathrm{s} \mathrm{K})$. The presented results are in very good agreement with the reference data from [71]. A larger and more quantitative study has been performed over the last months using several other mixtures of the Fointainebleu benchmark and an extended paper containing all the data sets has been published recently [72].

In summary, the method outlined in this manuscript is capable of providing a measurement of the main mass transport properties of the analyzed fluid mixture without previous knowledge of the so-called optical contrast factors of the mixture $\partial n / \partial T$ and $\partial n / \partial c$. To our current knowledge, the latter are required for any other optical characterization technique. Another experimental advantage of our approach is that the sample thickness can be chosen as small as needed, provided that a sufficient optical contrast is obtained. As a rule of thumb, one should satisfy the condition $S_{T} \times h>1 \times 10^{-4} \mathrm{~cm} / \mathrm{K}$. This can be a significant advantage for measurements on mixtures containing colloidal particles, where Soret coefficients are typically 10 (or more) times larger than for molecular mixtures. In that case, one can design a cell 10 times thinner, thus providing $10^{3}$ times shorter decay times, which can compensate the typically smaller diffusion coefficients. Moreover, this method allows direct investigation of non-equilibrium fluctuations, which are intimately related to the nature of the mass transport processes [73].

\section{Conclusions}

Here we have presented a preliminary analysis of the static and the dynamic power spectra of non-equilibrium fluctuations of a binary liquid mixture under thermal stress. Our attention has been directed towards the wave vector dependent time decay as well as the value of the critical size dictated by the interaction between the gravitational force and nonequilibrium fluctuations. Such a measurement is able to provide quantitative information about the diffusion and Soret coefficients of binary mixtures in the case of a positive Soret coefficient. The evaluation of these quantities is made possible thanks to a non-invasive optical method, namely dynamic near-field scattering with a shadowgraph setup and a differential dynamic analysis.

\section{Acknowledgements}

F.C. is grateful to Laura Chantada for help with the experimental setup and to Giovanni Cerchiari for help with the analysis software. F.C. acknowledges financial support from the European Union under Marie Curie funding, contract No. IEF-251131, DyNeFI Project.

\section{References}

[1] C. Ludwig, Sitz.-ber. Akad. Wiss. Wien, Math.-Nat. Kl. 20 (1856) 539.

[2] C. Soret, Arch. Sci. 3 (1879) 48.

[3] S. Hartmann, W. Köhler, K.I. Morozov, Soft Matter 8 (2012) 1355.

[4] S. Hartmann, G. Wittko, W. Köhler, K.I. Morozov, K. Albers, G. Sadowski, Phys. Rev. Lett. 109 (2012) 065901.

[5] K.I. Morozov, Phys. Rev. E 79 (2009) 031204.

[6] S. Guillain-Giullot, A. Würger, Phys. Rev. E 83 (2011) 03501(R).

[7] A. Würger, Phys. Rev. Lett. 102 (2009) 078302.

[8] P.-A. Artola, B. Rousseau, Phys. Rev. Lett. 98 (2007) 125901.

[9] G. Galliero, S. Volz, J. Chem. Phys. 128 (2008) 064505.

[10] G. Galliero, B. Duguay, J.-P. Caltagirone, F. Montel, Philos. Mag. 83 (2003) 2097.

[11] F. Bresme, A. Lervik, D. Bedeaux, S. Kjelstrup, Phys. Rev. Lett. 101 (2008) 020602.

[12] M. Yang, M. Ripoll, J. Phys.: Condens. Matter 24 (2012) 195101. 
[13] R. Piazza, A. Guarino, Phys. Rev. Lett. 88 (2002) 208302.

[14] M. Braibanti, D. Vigolo, R. Piazza, Phys. Rev. Lett. 100 (2008) 108303.

15] S. Duhr, D. Braun, Proc. Natl. Acad. Sci. USA 103 (2006) 19678.

[16] S. Duhr, D. Braun, Phys. Rev. Lett. 96 (2006) 168301.

17] S. Wiegand, J. Phys.: Condens. Matter 16 (2004) R357.

[18] J.K. Platten, M.M. Bou-Ali, P. Blanco, J.A. Madariaga, C. Santamaria, J. Phys. Chem. B 111 (2007) 11524.

[19] V. Shetsova, A. Mialdun, D. Melnikov, I. Ryzhkov, Y. Gaponenko, M.Z. Saghir, T. Lyubimova, J.-C. Legros, C. R. Mec. 339 (2011) 310.

[20] A. Mialdun, V. Yasnou, V. Shevtsova, A. Königer, W. Köhler, D. Alonso de Mezquia, M.M. Bou-Ali, J. Chem. Phys. 136 (2012) 244512.

[21] S. Van Vaerenbergh, J.-C. Legros, J. Phys. Chem. B 102 (1998) 4426.

[22] M. Touzet, G. Galliero, V. Lazzeri, M.Z. Saghir, F. Montel, J.-C. Legros, C. R. Mec. 339 (2011) 318.

23] S. Van Vaerenbergh, S. Srinivasan, M.Z. Saghir, J. Chem. Phys. 131 (2009) 114505.

[24] M. Giglio, A. Vendramini, Phys. Rev. Lett. 34 (1975) 561.

[25] M. Giglio, A. Vendramini, Phys. Rev. Lett. 38 (1977) 26.

[26] S. Wongsuwarn, D. Vigolo, R. Cerbino, A.M. Howe, A. Vailati, R. Piazza, P. Cicuta, Soft Matter 8 (2012) 5857.

[27] F. Croccolo, M.A. Arnaud, D. Bégué, H. Bataller, J. Chem. Phys. 135 (2011) 034901.

[28] F. Croccolo, F. Plantier, G. Galliero, G. Pijaudier-Cabot, M.Z. Saghir, F. Dubois, S. Van Vaerenbergh, F. Montel, H. Bataller, Rev. Sci. Instrum. 82 (2011) 126105.

[29] J.K. Platten, M.M. Bou-Ali, J.-F. Dutrieux, Phil. Mag. 83 (2003) 2001.

[30] A. Mialdun, V.M. Shevtsova, J. Chem. Phys. 134 (2011) 044524.

[31] W. Köhler, R. Schäfer, Adv. Polym. Sci. 151 (2000) 1.

[32] M. Giglio, A. Vendramini, Appl. Phys. Lett. 25 (1974) 555.

[33] F. Croccolo, H. Bataller, G. Pijaudier-Cabot, AIP Conf. Proc. 1254 (2010) 157.

[34] W. Köhler, A. Krekhov, W. Zimmermann, Adv. Polym. Sci. 227 (2010) 145.

[35] J.F. Torres, A. Komiya, E. Shoji, J. Okajima, S. Maruyama, Opt. Las. Eng. 50 (2012) 1287.

36] M. Giglio, M. Carpineti, A. Vailati, Phys. Rev. Lett. 85 (2000) 1416.

[37] D. Brogioli, A. Vailati, M. Giglio, Appl. Phys. Lett. 81 (2002) 4109.

[38] F. Ferri, D. Magatti, D. Pescini, M.A.C. Potenza, M. Giglio, Phys. Rev. E 70 (2004) 041405.

[39] R. Cerbino, A. Vailati, Curr. Opin. Colloid Interf. Sci. 14 (2009) 416.

[40] R. Cerbino, F. Scheffold, Curr. Opin. Colloid Interf. Sci. 12 (2007) 50.

[41] D. Brogioli, F. Croccolo, V. Cassina, D. Salerno, F. Mantegazza, Opt. Expr. 16 (2008) 20272-20282.

[42] D. Brogioli, V. Cassina, D. Salerno, F. Croccolo, F. Mantegazza, Opt. Expr. 17 (2009) 1222-1233.

[43] D. Brogioli, D. Salerno, V. Cassina, F. Mantegazza, Opt. Expr. 17 (2009) 15431-15448.

[44] D. Salerno, D. Brogioli, F. Croccolo, R. Ziano, F. Mantegazza, Opt. Expr. 19 (2011) 26416-26422.

[45] D. Brogioli, D. Salerno, F. Croccolo, R. Ziano, F. Mantegazza, New J. Phys. 13 (2011) 123007.

[46] F. Croccolo, D. Brogioli, A. Vailati, M. Giglio, D.S. Cannell, Ann. N.Y. Acad. Sci. 1077 (2006) 365-379.

[47] F. Croccolo, D. Brogioli, A. Vailati, M. Giglio, D.S. Cannell, Phys. Rev. E 76 (2007) 41112.

[48] F. Croccolo, D. Brogioli, Appl. Opt. 50 (2011) 3419-3427.

[49] F. Croccolo, D. Brogioli, A. Vailati, M. Giglio, D.S. Cannell, Appl. Opt. 45 (2006) 2166-2173.

[50] G. Cerchiari, F. Croccolo, F. Cardinaux, F. Scheffold, Rev. Sci. Instrum. 83 (2012) 106101.

[51] R. Cerbino, V. Trappe, Phys. Rev. Lett. 100 (2008) 188102.

[52] D. Magatti, M.D. Alaimo, M.A.C. Potenza, F. Ferri, Appl. Phys. Lett. 92 (2008) 241101.

[53] S.P. Trainoff, D.S. Cannell, Phys. Fluids 14 (2002) 1340.

[54] G.S. Settles, Schlieren and Shadowgraph Techniques: Visualizing Phenomena in Transparent Media, Springer-Verlag, Berlin, Heidelberg, New York, 2001.

[55] F. Giavazzi, D. Brogioli, V. Trappe, T. Bellini, R. Cerbino, Phys. Rev. E 80 (2009) 031403.

[56] L.G. Wilson, V.A. Martinez, J. Schwarz-Linek, J. Tailleur, G. Bryant, P.N. Pusey, W.C.K. Poon, Phys. Rev. Lett. 106 (2011) 018101.

[57] M. Reufer, V.A. Martinez, P. Schurtenberger, W.C.K. Poon, Langmuir 28 (2012) 4618-4624.

[58] P.J. Lu, F. Giavazzi, T.E. Angelini, E. Zaccarelli, F. Jargstorff, A.B. Schofield, J.N. Wilking, M.B. Romanowsky, D.A. Weitz, R. Cerbino, Phys. Rev. Lett. 108 (2012) 218103

[59] P.N. Segré, J.V. Sengers, Phys. A 198 (1993) 46

[60] W.B. Li, P.N. Segré, R.W. Gammon, J.V. Sengers, M. Lamvik, J. Chem. Phys. 101 (1994) 5058.

[61] K.J. Zhang, M.E. Briggs, R.W. Gammon, J.V. Sengers, J. Chem. Phys. 104 (1996) 6881.

[62] J.M. Ortiz de Zarate, J.V. Sengers, Hydrodynamic Fluctuations in Fluids and Fluid Mixtures, Elsevier Science, New York, 2006.

[63] A. Vailati, M. Giglio, Phys. Rev. E 58 (1998) 4361.

[64] A. Vailati, M. Giglio, Phys. Rev. Lett. 77 (1996) 1484.

65] A. Vailati, M. Giglio, Nature 390 (1997) 262.

[66] D. Brogioli, A. Vailati, M. Giglio, Phys. Rev. E 61 (2000) R1.

[67] A. Oprisan, S. Oprisan, A. Teklu, Appl. Opt. 49 (2010) 86-98.

[68] A. Vailati, R. Cerbino, S. Mazzoni, C.J. Takacs, D.S. Cannell, M. Giglio, Nat. Commun. 2 (2011) 290

[69] C.J. Takacs, A. Vailati, R. Cerbino, S. Mazzoni, M. Giglio, D.S. Cannell, Phys. Rev. Lett. 106 (2011) 244502.

[70] F. Croccolo, H. Bataller, F. Scheffold, in preparation.

[71] J.K. Platten, M.M. Bou-Ali, P. Costesèque, J.-F. Dutrieux, W. Köhler, C. Leppla, S. Wiegand, G. Wittko, Phil. Mag. 83 (2003) 1965.

[72] F. Croccolo, H. Bataller, F. Scheffold, J. Chem. Phys. 137 (2012) 234202.

[73] D. Brogioli, A. Vailati, Phys. Rev. E 63 (2000) 012105. 